\title{
Doubly Coexisting Dark Matter Candidates in an Extended Seesaw Model
}

\author{
Sin Kyu Kang ${ }^{1, *}$, H. Sung Cheon ${ }^{2, \dagger}$, C. S. Kim ${ }^{2, \ddagger}$ \\ 1 School of Liberal Arts, Seoul National University of Technology, Seoul 121-742, Korea \\ 2 Dept. of Physics and IPAP, Yonsei University, Seoul 120-749, Korea
}

\begin{abstract}
We examine how a scenario of coexisting two-particle dark mater can be realized in the extended seesaw model, which we have proposed previously to accommodate small neutrino masses and low scale leptogenesis with an introduction of singlet Majorana neutrino $S$ and singlet scalar $\phi$. We now impose the discrete symmetry $Z_{2} \times Z_{2}^{\prime}$ and introduce new renormalizable interaction terms with a new heavy singlet scalar particle $\Phi$ so as for previously introduced $S$ and $\phi$ to be doubly coexisting dark matter candidates. Depending on the mass spectrum of the two dark matter candidates, the annihilation process either $S S \longrightarrow \phi \phi$ or $\phi \phi \longrightarrow S S$ is of particular interest because the annihilation cross sections for the processes can be so large that the relic abundance of decaying particle should get lowered, which in turn makes the constraints on its parameter space relaxed, compared with the case of one and only one dark matter candidate. We discuss the implications of the dark matter detection through the scattering off the nucleus of the detecting material on our scenarios for dark matter candidates. We also study the implications for the search of invisible Higgs decay at LHC, which may serve as a probe of our scenario for dark matter.
\end{abstract}

PACS numbers: 98.80.-k, 95.35.+d, 14.60.St, 14.80.Cp

\footnotetext{
*E-mail: skkang@snut.ac.kr

$\dagger$ E-mail: hscheon@gmail.com

$\ddagger$ E-mail: cskim@yonsei.ac.kr
} 


\section{INTRODUCTION}

To identify the nature of dark matter remains an open question in particle physics and cosmology. The amount of cold dark matter in the Universe, which has been determined precisely from 5 year WMAP data [1], is given by $\Omega_{\mathrm{CDM}} h^{2}=0.1099 \pm 0.0062$. None of the SM particles can be a good candidate for the dark matter, so that the existence of the dark matter itself points to new physics beyond the Standard Model (SM). It has been argued that weakly interacting massive particles (WIMPs) are promising dark matter candidates among various proposals in the literature [2, 3]. With the appropriate size of its interactions, natural values of its mass around the $\mathrm{TeV}$ scale and annihilation cross-section into the SM particles, the observed cosmological abundance is suitably accounted for by the WIMP relic density [3, 4]. This weak-scale annihilation cross-section, when reversed, properly suggests a weak-scale production cross-section at colliders, and, when viewed in the $t$-channel, implies an elastic scattering cross-section on nuclei is within reach of purpose-built underground detectors. In recent years, experiments of the latter type have reached an impressive level of sensitivity [5, 6], and significant future progress in this direction is anticipated.

While dark matter is usually assumed to have one and only one particle candidate, a possibility of multiply coexisting dark matter particles has been recently explored [7] (There has been an attempt to demonstrate the total amount of dark matter with a composition of $\mathrm{keV}$ sterile neutrinos and a generic CDM [8].) The simplest generic scenario of the multi-particle dark matter is to have coexisting two particles as dark matter candidates by imposing discrete symmetry $Z_{2} \times Z_{2}^{\prime}$ in the Lagrangian. As discussed in Ref. [7], the scenario can be naturally realized in the minimal supersymmetric Standard Model. One of virtues of such a scenario is that severe constraints on the model parameter space can be relaxed due to the presence of the second dark matter particle.

Recently, we proposed a model to accommodate low scale leptogenesis, tiny neutrino masses and the existence of dark matter [9]. Our aim has been accomplished by introducing new gauge singlet neutrinos and a singlet scalar boson on top of the SM particles and the right handed singlet Majorana neutrinos [10]. Imposing discrete $Z_{2}$ symmetry in the model, either the newly introduced singlet neutrino or the singlet scalar boson could be a dark matter candidate depending on their mass spectrum under the assumption that dark matter is one and only one particle candidate as usual [11, 12, 13]. Motivated by the work [7], in 
this paper, we examine if the new singlet neutrino and the singlet scalar boson in the model can be coexisting two-particle dark matter (2DM) candidates abandoning the assumption on only one dark matter. In fact, as discussed in [9], when the new singlet neutrino in the model we proposed is the only one dark matter candidate, the co-annihilation process [14] is responsible for the relic abundance required for dark matter and the allowed parameter space for achieving the right amount of the relic abundance has been determined to be very narrow. Thus, it would be interesting to investigate whether the 2DM scenario realized in our model can make the allowed parameter space relaxed or not. In this scenario, we introduce new renoramlizable interaction terms with a new heavy singlet scalar particle $\Phi$ so as for previously introduced $S$ and $\phi$ to be doubly coexisting dark matter candidates. As will be discussed later, the important point deserved to notice is that such scenario may open up a new annihilation process of the heavier dark matters into the lighter ones, which dominantly contributes to the relic abundance and thus plays a crucial role in making the allowed parameter space relaxed.

This paper is organized as follows: In Section II, we show how the scenario of coexisting 2DM candidates can be realized in the model we previously proposed. To achieve our goal, we introduce new renormalizable interaction terms among the SM Higgs boson, the heavy singlet scalar, the singlet neutrino and the singlet scalar boson in the Lagrangian. In Section III, we investigate the relic abundance and present the allowed regions of the parameter space for the possible coexisting 2DM candidates. In Section IV, we discuss about dark matter detection through the scattering off the nucleus of the detecting material. In Section V, we study how we can probe the coexisting 2DM candidates through the search for the Higgs decay at collider experiment, particularly at LHC.

\section{COEXISTING TWO-PARTICLE DARK MATTER CANDIDATES}

To accommodate low scale leptogenesis, tiny neutrino masses and dark matter, the model we proposed in the previous work [9] is described by the following Lagrangian,

$$
\begin{aligned}
\mathcal{L}_{\text {Ref. [9] }}= & \mathcal{L}_{\mathrm{SM}}+\left(Y_{D} \bar{\nu} H N+Y_{S} \bar{N} \phi S+\text { h.c. }\right)+M_{R} N^{T} N-m_{S^{0}} S^{T} S \\
& +\frac{1}{2}\left(\partial_{\mu} \phi\right)^{2}-\frac{1}{2} m_{\phi^{0}}^{2} \phi^{2}-\frac{\lambda_{s}}{4} \phi^{4}-\lambda H^{\dagger} H \phi^{2},
\end{aligned}
$$


where the first term is the Lagrangian of the $\mathrm{SM}$ and $\nu, N, S$ stand for $S U(2)_{L}$ doublet, right-handed singlet and singlet Majorana neutrinos, respectively. $H$ and $\phi$ denote the $S U(2)_{L}$ doublet and singlet scalar fields. As discussed in [9], the newly introduced neutral particle, either singlet Majorana neutrino or singlet scalar boson, can be a candidate for dark matter, provided that one imposes $Z_{2}$ symmetry under which $S$ and $\phi$ are odd and all other particles even. In this model, low scale leptogenesis of order 1-10 TeV can be achieved when we take, for example, $Y_{D} \sim 10^{-6}$ and $Y_{S} \sim 10^{-3}[10]$. Due to such small Yukawa couplings, the cross section for the annihilation of $S$ into a pair of $\phi$ is too small, so that the coannihilation processes are compulsory to achieve the right amount of the relic abundance, which in turn lead to a bit tight constraints on the parameter space [9].

Now, let us extend the model described by the Lagrangian Eq. (11) so that the scenario of the coexisting 2DM can be realized. In order to guarantee the stability of the 2DM candidates, we first impose the discrete symmetry $Z_{2} \times Z_{2}^{\prime}$ under which all the SM particles are $(+,+)$, singlet neutrino $S$ is $\sim(-,+)$ and the singlet scalar boson $\phi$ is $\sim(+,-)$. In addition to the Lagrangian Eq. (1), we introduce new renormalizable terms given by

$$
\begin{aligned}
\mathcal{L}= & \mathcal{L}_{\text {Ref. }[9]}+Y_{\Phi} \bar{S} \Phi S+\frac{1}{2} m_{H^{0}}^{2} H^{\dagger} H-\frac{\lambda_{1}}{4} H^{\dagger} H H^{\dagger} H+\frac{1}{2} m_{\Phi^{0}}^{2} \Phi^{2} \\
- & \frac{\lambda_{2}}{4} \Phi^{4}-\lambda_{3} \phi^{2} \Phi^{2}-\lambda_{4} H^{\dagger} H \Phi^{2}
\end{aligned}
$$

where $\Phi$ is the SM-like $(+,+)$ heavy singlet scalar particle, whose mass is assumed to be larger than those of $\phi$ and $S$. Here, we demand that the minimum of the scalar potential is bounded from below so as to guarantee the existence of vacuum and the minimum of the scalar potential must spontaneously break the electroweak gauge group, $\left\langle H^{0}\right\rangle,\langle\Phi\rangle \neq 0$, but must not break $Z_{2} \times Z_{2}^{\prime}$ symmetry imposed above. After spontaneous symmetry breaking, the part of the scalar potential is given by

$$
\begin{aligned}
V= & \frac{1}{2} m_{\phi}^{2} \phi^{2}-\frac{1}{2} m_{h}^{2} h^{2}-\frac{1}{2} m_{\Phi}^{2} \Phi^{2}+2 \lambda_{4} v_{h} v_{\Phi} h \Phi+\frac{\lambda_{s}}{4} \phi^{4}+\frac{\lambda_{1}}{4} v_{h} h^{3}+\frac{\lambda_{1}}{16} h^{4} \\
& +\frac{\lambda_{2}}{4} \Phi^{4}+\lambda_{2} v_{\Phi} \Phi^{3}+\frac{\lambda}{2} \phi^{2} h^{2}+\lambda v_{h} \phi^{2}+\lambda_{3} \phi^{2} \Phi^{2}+2 \lambda_{3} v_{\Phi} \Phi \phi^{2} \\
& +\frac{\lambda_{4}}{2} h^{2} \Phi^{2}+\lambda_{4} v_{\Phi} h^{2} \Phi+\lambda_{4} v_{h} \Phi^{2}+h . c .
\end{aligned}
$$


where

$$
\begin{aligned}
m_{\phi}^{2} & =m_{\phi^{0}}^{2}+\lambda v_{h}^{2}+2 \lambda_{3} v_{\Phi}^{2} \\
m_{h}^{2} & =\frac{1}{2} m_{H^{0}}^{2}-\frac{3}{4} \lambda_{1} v_{h}^{2}-\lambda_{4} v_{\Phi}^{2} \\
m_{\Phi}^{2} & =m_{\Phi^{0}}^{2}-3 \lambda_{2} v_{\Phi}^{2}-\lambda_{4} v_{h}^{2}
\end{aligned}
$$

Here, we have adopted $\sqrt{2} H^{T}=(h, 0)$ and shifted the Higgs boson $h$ and the singlet scalar $\Phi$ by $h \rightarrow h+v_{h}$ and $\Phi \rightarrow \Phi+v_{\Phi}$, respectively. The relevant size of $v_{\Phi}$ in this work is of order $1 \mathrm{TeV}$. Since there exists a mixing mass term between $h$ and $\Phi$, we rotate them with $\Phi=-s h^{\prime}+c \Phi^{\prime}$ and $h=c h^{\prime}+s \Phi^{\prime}$, where $s$ and $c$ are $\sin \theta$ and $\cos \theta$, respectively. Then, the effective potential is written as

$$
\begin{aligned}
V_{e f f}= & \frac{m_{\phi}^{\prime 2}}{2} \phi^{2}+\frac{m_{h}^{\prime 2}}{2} h^{\prime 2}+\frac{m_{\Phi}^{\prime 2}}{2} \Phi^{\prime 2}+\frac{\lambda_{4}}{2} h^{\prime 2} \Phi^{\prime 2} \\
& +\frac{1}{2}\left(\lambda c^{2}+2 \lambda_{3} s^{2}\right) \phi^{2} h^{\prime 2}+\left(\frac{\lambda}{2} s^{2}+\lambda_{3} c^{2}\right) \phi^{2} \Phi^{\prime 2} \\
& +\kappa_{1} h^{\prime 3}+\kappa_{2} \Phi^{\prime 3}+\alpha \Phi^{\prime} \phi^{2}+\beta h^{\prime 2} \Phi^{\prime}+\gamma h^{\prime} \phi^{2} \\
& +\left(\frac{\lambda_{1}}{16} c^{4}+\frac{\lambda_{4}}{2} c^{2} s^{2}+\frac{\lambda_{2}}{4} s^{4}\right) h^{\prime 4}+\left(\frac{\lambda_{2}}{4} c^{4}+\frac{\lambda_{4}}{2} c^{2} s^{2}+\frac{\lambda_{1}}{16} s^{4}\right) \Phi^{\prime 4}
\end{aligned}
$$

where

$$
\begin{aligned}
\kappa_{1} & =\lambda_{4} c s\left(c v_{\Phi}+s v_{h}\right)+\frac{\lambda_{1}}{4} c^{3} v_{h}+\lambda_{2} v_{\Phi} s^{3} \\
\kappa_{2} & =\lambda_{2} c^{3} v_{\Phi}-\lambda_{4} c s\left(c v_{h}-s v_{\Phi}\right)-\frac{\lambda_{1}}{4} s^{3} v_{h} \\
\alpha & =-\lambda v_{h} s+2 \lambda_{3} v_{\Phi} c \\
\beta & =\lambda_{4}\left[v_{\Phi} c^{3}-v_{h} s^{3}+2 s c\left(s v_{\Phi}-c v_{h}\right)\right]+c s\left[-\frac{3}{4} \lambda_{1} v_{h} c+3 \lambda_{2} v_{\Phi} s\right] \\
\gamma & =2 \lambda_{3} s v_{\Phi}+\lambda v_{h} c .
\end{aligned}
$$

On the other hand, the Lagrangian containing the field $S$ becomes,

$$
\mathcal{L}_{S}=-m_{S} S^{T} S+\left(s Y_{\Phi}\right) h^{\prime} S^{T} S+\left(c Y_{\Phi}\right) \Phi^{\prime} S^{T} S
$$

where $m_{S}=\left(m_{S^{0}}-Y_{\Phi} v_{\Phi}\right)$ is the physical mass of $\mathrm{S}$.

For our purpose, in this work, we keep the new coupling constants $Y_{\Phi}, \lambda_{i(i=1, . ., 4)}$ to be nonzero. For $m_{S} \gtrsim m_{\phi}$, we see from the interaction terms in Eqs. (5, 17) that the singlet neutrino $S$ can annihilate into $\phi \phi$ and $h h$, in addition to the ordinary particles. The produced scalar field $\phi$ from the annihilation of $S$ decays into the SM particles. The annihilation process, 
$S S \rightarrow \phi \phi$, is of particular interest because the annihilation cross section for this process can be so large that $S$ could predominantly annihilate into $\phi \phi$, resulting in a much smaller relic

abundance, thereby relaxing the constraints on its parameter space as expected. In fact, the annihilation of $S$ into $\phi \phi$ also occurs in the scenario with only one dark matter candidate proposed in [9], but its contribution to the relic abundance can not work because both $S$ and $\phi$ are odd particles under $Z_{2}$ symmetry. Thus, the enhancement of the annihilation rate for the process $S S \rightarrow \phi \phi$ is a distinctive feature of the $2 \mathrm{DM}$ scenario. For $m_{\phi} \gtrsim m_{S}$, the conclusions are mostly the same if we switch $S$ and $\phi$.

\section{RELIC ABUNDANCE IN TWO-PARTICLE DARK MATTER SCENARIO}

Assuming the coexistence of two dark matter candidates, the relic abundance observed must be composed of the contributions of both $S$ and $\phi$ as follows,

$$
\Omega_{S} h^{2}+\Omega_{\phi} h^{2}=\Omega_{\mathrm{CDM}} h^{2}=0.110 \pm 0.006 .
$$

The relic density of each dark matter species is approximately given by

$$
\Omega_{i} h^{2} \approx(0.1 p b) /<\sigma v>_{i} \quad(i=S, \phi),
$$

where $\langle\sigma v\rangle_{i}$ is the thermally averaged product of its annihilation cross section with its velocity. For our convenience, we define the parameter $\varepsilon_{i}$ as a ratio of $\Omega_{i} h^{2}$ to $\Omega_{\mathrm{CDM}} h^{2}$,

$$
\varepsilon_{i}=\frac{\Omega_{i} h^{2}}{\Omega_{\mathrm{CDM}} h^{2}},
$$

where $\varepsilon_{S}+\varepsilon_{\phi}=1$. In fact, the parameter $\varepsilon_{i}$ represents the fraction of the mass density of each dark matter species in our local dark-matter halo as well as in the Universe. Since the values of $\varepsilon_{i}$ are unknown, we consider a few cases by choosing their values in the analysis.

To calculate $\Omega_{i} h^{2}$, the input parameters to be fixed are the SM Higgs mass $m_{h}$ and masses of $m_{S}, m_{\phi}$ and $m_{\Phi}$, the vacuum expectation values $v_{h}$ and $v_{\Phi}$, mixing angle $\theta$ and the coupling constants $Y_{\Phi}, \lambda, \lambda_{i(i=1, \ldots, 4)}$. First of all, varying the two parameters $Y_{\Phi}$ and $m_{S}$ while fixing all others, we calculate each $\Omega_{i} h^{2}$ with the help of the micrOMEGAs 2.0.7 program [15], and then pick up the parameter space $Y_{\Phi}$ and $m_{S}$ resulting in the chosen value of $\varepsilon_{S}$.

In Fig. 1, we present the correlations between $Y_{\Phi}$ and $m_{S}$ for (a) $m_{\phi}=350 \mathrm{GeV}$ and $\varepsilon_{s}=0.8$, (b) $m_{\phi}=74 \mathrm{GeV}$ and $\varepsilon_{s}=0.8$, (c) $m_{\phi}=250 \mathrm{GeV}$ and $\varepsilon_{s}=0.9$, (d) $m_{\phi}=74 \mathrm{GeV}$ 

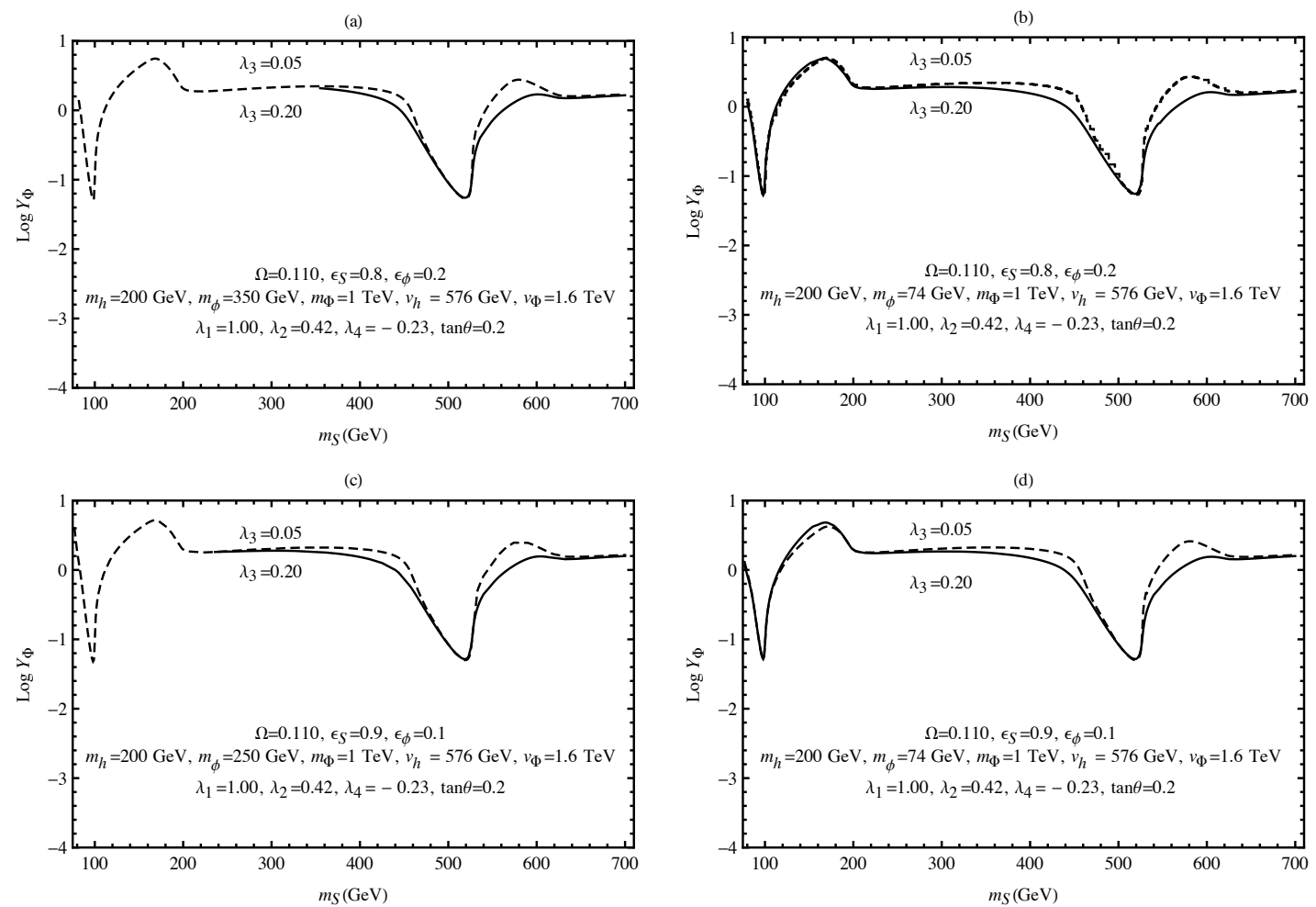

FIG. 1: Plots of $Y_{\Phi}$ as a function of $m_{S}$ for $\Omega_{\mathrm{CDM}} h^{2}=0.110$. We take $m_{h}=200 \mathrm{GeV}$. The panels correspond to (a) $m_{\phi}=350 \mathrm{GeV}, \varepsilon_{S}=0.8, \varepsilon_{\phi}=0.2$, (b) $m_{\phi}=74 \mathrm{GeV}, \varepsilon_{S}=0.8, \varepsilon_{\phi}=0.2$, (c) $m_{\phi}=250 \mathrm{GeV}, \varepsilon_{S}=0.9, \varepsilon_{\phi}=0.1$, (d) $m_{\phi}=74 \mathrm{GeV}, \varepsilon_{S}=0.9, \varepsilon_{\phi}=0.1$. The dashed (solid) lines correspond to $\lambda_{3}=0.05(0.20)$.

and $\varepsilon_{s}=0.9$. The solid and dashed lines correspond to $\lambda_{3}=0.20, \lambda_{3}=0.05$, respectively. Here, we take the value of $\Omega_{\mathrm{CDM}} h^{2}$ to be 0.110 , and the input values of the other parameters are given by $m_{h}=200 \mathrm{GeV}, m_{\Phi}=1 \mathrm{TeV}, \lambda_{1}=1.0, \lambda_{2}=0.42, \lambda_{4}=-0.23, v_{h}=576 \mathrm{GeV}$, $v_{\Phi}=1.6 \mathrm{TeV}$ and $\tan \theta=0.2$. For the regime $m_{\phi} \lesssim m_{S}$, the relic abundance of the field $S$ depends on the annihilation of $S$ into the singlet scalar $\phi$ and the SM particles whose annihilation cross sections contain the couplings $\lambda, \lambda_{i(i=1, \ldots, 4)}, Y_{\Phi}$ as well as the SM Yukawa couplings. In this case, the abundance of the singlet scalar field $\phi$ depends mainly on the annihilation processes of $\phi$ into the SM particles whose annihilation cross section contains the coupling $\lambda, \lambda_{3}$ and the SM Yukawa couplings. Thus, the value of $\lambda$ is determined from the chosen values of $\varepsilon_{\phi}$ and $\lambda_{3}$. Once $\lambda$ is fixed in this way, we can obtain the correlation between $Y_{\Phi}$ and $m_{S}$ for a fixed value of $\lambda_{3}$ as presented in Fig. 1 by fitting to the chosen value of $\varepsilon_{S}$. On the other hand, for the regime $m_{S} \lesssim m_{\phi}$, the relic abundance of $S$ depends mainly on 

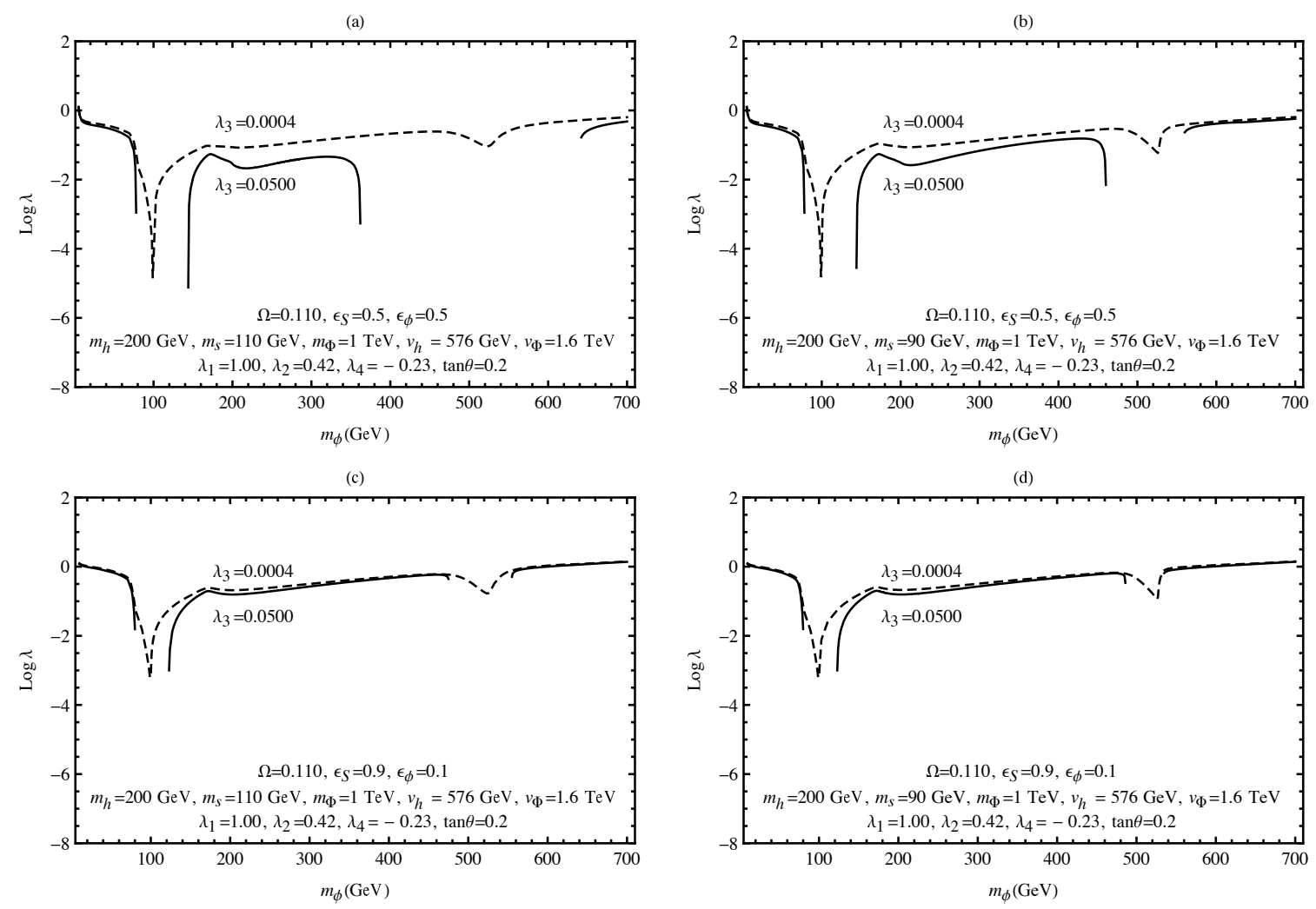

FIG. 2: Plots of $\lambda$ as a function of $m_{\phi}$ for $\Omega_{\mathrm{CDM}} h^{2}=0.110$. We take $m_{h}=200 \mathrm{GeV}$ The panels correspond to (a) $m_{S}=110 \mathrm{GeV}, \varepsilon_{S}=0.5, \varepsilon_{\phi}=0.5$, (b) $m_{S}=90 \mathrm{GeV}, \varepsilon_{S}=0.5, \varepsilon_{\phi}=0.5$, (c) $m_{S}=110 \mathrm{GeV}, \varepsilon_{S}=0.9, \varepsilon_{\phi}=0.1$ and $(\mathrm{d}) m_{S}=90 \mathrm{GeV}, \varepsilon_{S}=0.9, \varepsilon_{\phi}=0.1$. The dashed (solid) lines represent $\lambda_{3}=0.0004(0.0500)$.

the annihilation process of $S$ into the SM particles whose annihilation cross sections contain the coupling $Y_{\Phi}$ and the SM Yukawa couplings. Thus, the value of $Y_{\Phi}$ as a function of $m_{S}$ is determined from the chosen value of $\varepsilon_{S}$ irrespective of the value of $\lambda_{3}$. We see from Fig. 1 that the region $m_{S} \lesssim m_{\phi}$ is not allowed in the case of $\lambda_{3}=0.2$. This is because the rate of the annihilation of $\phi$ into $S S$ becomes large for $m_{S} \lesssim m_{\phi}$ and large value of $\lambda_{3}$, which makes $\varepsilon_{\phi}$ lower for achieving the required relic abundance.

In Fig. 2, we present the correlation between $\lambda$ and $m_{\phi}$ for $\Omega_{\mathrm{CDM}} h^{2}=0.110$ and (a) $m_{S}=110 \mathrm{GeV}$ and $\varepsilon_{S}=0.5$, (b) $m_{S}=90 \mathrm{GeV}$ and $\varepsilon_{S}=0.5$, (c) $m_{S}=110 \mathrm{GeV}$ and $\varepsilon_{S}=0.9$, and $(\mathrm{d}) m_{S}=90 \mathrm{GeV}$ and $\varepsilon_{S}=0.9$. The solid (dashed) lines correspond to $\lambda_{3}=0.0500$ (0.0004). The values of the input parameters $m_{h}, m_{\Phi}, v_{h}, v_{\Phi}, \theta, \lambda_{1}, \lambda_{2}, \lambda_{4}$ are the same as those in Fig. 1. For the regime $m_{S} \lesssim m_{\phi}$, the relic abundance of the field $\phi$ depends on the annihilation of $\phi$ into the singlet fermion $S$ and the SM particles whose annihilation 
cross sections contain the couplings $\lambda, \lambda_{i(i=1, \ldots, 4)}, Y_{\Phi}$ as well as the SM Yukawa couplings. In this case, the abundance of the singlet neutrino $S$ depends on the annihilation processes of $S$ into the SM particles whose annihilation cross section contains the coupling $Y_{\Phi}$ and the SM Yukawa couplings. Thus, the value of $Y_{\Phi}$ is first determined from a chosen value of $\varepsilon_{S}$, and then we obtain the correlation between $\lambda$ and $m_{\phi}$ for a fixed value of $\lambda_{3}$, as presented in Fig. 2 by fitting to the chosen value of $\varepsilon_{\phi}$. As one can see from Fig. 2, there exist two disconnected regions of $m_{\phi}$ in each panels for $\lambda_{3}=0.05$ : one is around $2 m_{\phi} \simeq m_{h}$ corresponding to the SM higgs resonance region, and the other is around $2 m_{\phi} \simeq m_{\Phi}$ corresponding to the $\Phi$ resonance region, both of which are not allowed. On the other hand, for the regime $m_{\phi} \lesssim m_{S}$, the relic abundance of $\phi$ depends on the annihilation process of $\phi$ into the SM particles whose annihilation cross section contains the coupling $\lambda, \lambda_{3}$ and the SM Yukawa couplings. Thus, the value of $\lambda$ as a function of $m_{\phi}$ is determined from the chosen values of $\varepsilon_{\phi}$ and $\lambda_{3}$.

\section{IMPLICATION FOR DARK MATTER SEARCH}

To directly detect dark matter, typically proposed method is to detect the scattering of dark matter off the nucleus of the detecting material. Since the scattering cross section is expected to be very small, the energy deposited by a candidate for dark matter on the detector nucleus is also very small. In order to measure this small recoil energy, typically of order keV, of the nucleus, a very low threshold detector condition is required. Since the sensitivity of detectors to a dark matter candidate is controlled by their elastic scattering cross section with nucleus, it is instructive to examine how large the size of the elastic cross section could be. First, to estimate the elastic cross section with nucleus, we need to know the relevant matrix element for slowly moving spin-J nuclei, which is approximately given [13] by

$$
\frac{1}{2 J+1} \sum_{\text {spins }}\left|<n^{\prime}\right| \sum_{f} y_{f} \bar{f} f|n>|^{2} \simeq \frac{\left|A_{n}\right|^{2}}{(2 \pi)^{6}}
$$

where $n$ denotes nucleons and $\left|A_{n}\right|$ is determined to be

$$
\mathcal{A}_{n}=g_{h n n} \simeq \frac{190 \mathrm{MeV}}{v_{\mathrm{EW}}}
$$

by following the method given in [13] and taking the strange quark mass to be $95 \mathrm{MeV}$ and $<n|\bar{s} s| n>\sim 0.7$. 
Now, let us estimate the sizes of the elastic scattering cross sections in the scenario of the coexisting 2DM candidates, $S$ and $\phi$. The Feynman diagrams relevant to dark matternucleon scattering are presented in Fig. 3. The non-relativistic elastic scattering cross section for the singlet neutrino $S$-nucleon elastic scattering is given by

$$
\sigma_{S} \approx \frac{\left(Y_{\Phi} s\right)^{2}\left|\mathcal{A}_{n}\right|^{2}}{\pi}\left(\frac{m_{*}^{2}}{m_{h}^{4}}\right)
$$

where $m_{*}=m_{S} m_{n} /\left(m_{S}+m_{n}\right)$ is the reduced mass for the collision. Substituting Eq. (11) into Eq. (12), $\sigma_{S}$ (nucleon) becomes

$$
\sigma_{S}(\text { nucleon }) \approx \frac{1}{\pi}\left(\frac{Y_{\Phi} s \times 190 \mathrm{MeV}}{m_{h}^{2} v_{\mathrm{EW}}}\right)^{2}\left(\frac{m_{p} m_{S}}{m_{p}+m_{S}}\right)^{2},
$$

where the mass of $m_{p}$ is a mass of proton.

In the case of scalar $\phi$-nucleon elastic scattering, the non-relativistic elastic scattering cross section for $\phi$ is given by

$$
\sigma_{\phi}=\frac{\left(\lambda v_{h} c+2 \lambda_{3} s v_{\Phi}\right)^{2}\left|\mathcal{A}_{n}\right|^{2}}{4 \pi}\left(\frac{m_{*}^{2}}{m_{\phi}^{2} m_{h}^{4}}\right)
$$

where $m_{*}=m_{\phi} m_{n} /\left(m_{\phi}+m_{n}\right)$ is the reduced mass for the collision. Substituting (111) into Eq. (14), $\sigma_{\phi}$ (nucleon) becomes

$$
\sigma_{\phi}(\text { nucleon }) \approx \frac{1}{4 \pi}\left(\frac{\left(\lambda v_{h} c+2 \lambda_{3} s v_{\Phi}\right) 190 \mathrm{MeV}}{m_{h}^{2} v_{\mathrm{EW}}}\right)^{2}\left(\frac{m_{p}}{m_{p}+m_{\phi}}\right)^{2} .
$$

So far most experimental limits of the direct detection are given in terms of the scattering cross section per nucleon under the assumption that there exists only one dark matter

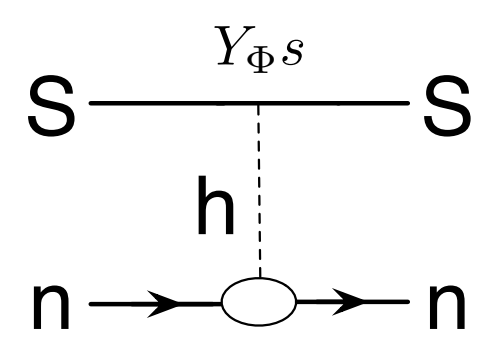

$g_{\text {hnn }}$

(a)

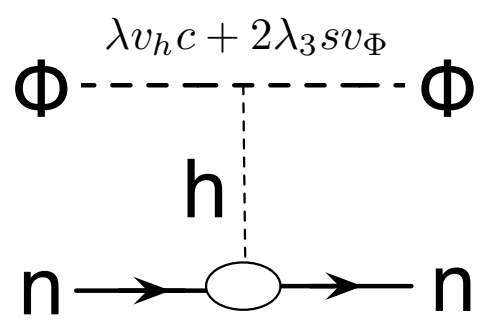

$g_{\text {hnn }}$

(b)

FIG. 3: Feynman diagrams relevant to (a) $S$-nucleon elastic scattering and (b) $\phi$-nucleon elastic scattering. 

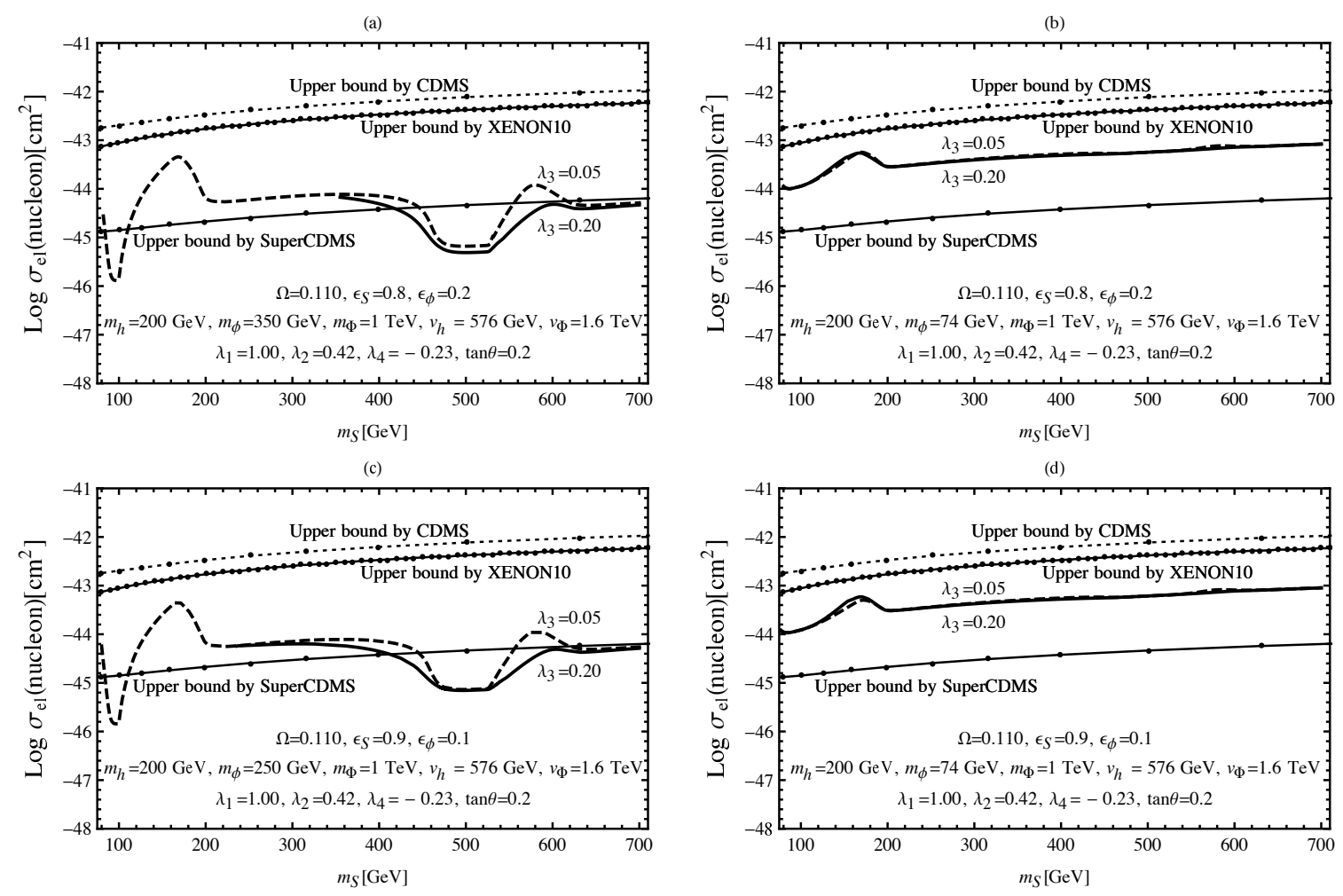

FIG. 4: Plots of the elastic cross section $\sigma_{e l}$ as a function of $m_{S}$ for $m_{h}=200 \mathrm{GeV}$ with $\varepsilon_{S}=0.8$, $\varepsilon_{\phi}=0.2$ and $(\mathrm{a}-\mathrm{b}) m_{\phi}=350(74) \mathrm{GeV}$; with $\varepsilon_{S}=0.9, \varepsilon_{\phi}=0.1$ and (c-d) $m_{\phi}=250$ (74) GeV. We present that the spin-independent WIMP-nucleon cross section upper limits (90\% C.L.) by XENON10 Dark Matter Experiment [16] and CDMS experiment [17]. In addition the expected reach of SuperCDMS collaboration [6] is presented.

candidate. In the scenario of 2DM, the cross section for the WIMP-nucleon elastic scattering $\sigma_{e l}$ is composed of the cross sections $\sigma_{S}$ and $\sigma_{\phi}[7]$;

$$
\frac{\sigma_{e l}}{m_{0}}=\frac{\varepsilon_{S}}{m_{S}} \sigma_{S}+\frac{\varepsilon_{\phi}}{m_{\phi}} \sigma_{\phi}
$$

where $m_{0}$ is the WIMP mass. Without loss of generality, we choose the singlet neutrino $S$ to be WIMP. In Fig. 4, we plot the predictions for $\sigma_{e l}$ as a function of $m_{S}$ for $m_{h}=200$ $\mathrm{GeV}$ in the cases of (a) $\varepsilon_{S}=0.8, \varepsilon_{\phi}=0.2, m_{\phi}=350 \mathrm{GeV}$, (b) $\varepsilon_{S}=0.8, \varepsilon_{\phi}=0.2, m_{\phi}=74$ $\mathrm{GeV}$, (c) $\varepsilon_{S}=0.9, \varepsilon_{\phi}=0.1, m_{\phi}=250 \mathrm{GeV}$, (d) $\varepsilon_{S}=0.9, \varepsilon_{\phi}=0.1, m_{\phi}=74 \mathrm{GeV}$.

On calculating $\sigma_{e l}$, we have used the correlation between $Y_{\Phi}$ and $m_{S}$ obtained in Fig. 1 corresponding to $\Omega=0.110$. Here, we also plot the curves for the new $90 \%$ C.L. upper bounds on the WIMP-nucleon spin-independent cross section as a function of WIMP mass obtained from XENON10 Dark Matter Experiment [16] and CDMS experiment [17]. In 
addition we present the expected reach of SuperCDMS collaboration [6]. While the current upper bounds on the spin-independent WIMP-nucleon cross sections by XENON10 and CDMS Dark Matter Experiments are not so strong to constrain the parameter space of the model, the expected reach of SuperCDMS experiment would at least strongly constrain it.

\section{IMPLICATION FOR HIGGS SEARCHES IN COLLIDER EXPERIMENTS}

Now we investigate the implications of our new scenario for Higgs searches in collider experiments. The singlet scalar boson $\phi$ and singlet fermion $S$ will not directly couple to ordinary matters, but only to the SM Higgs fields among the SM particles. Therefore, although the presence of the singlet particles will not affect electroweak phenomenology in a significant way, they will affect the phenomenology of the Higgs boson. In the scenario of only one dark matter candidate proposed in [9], the only possible channel to probe the scenario through the Higgs search is the invisible decay of the Higgs boson into $\phi \phi$. However, in the scenario of 2DM, the decay mode $h \rightarrow S S$ is also allowed due to new interaction terms [18]. The real Higgs boson can decay into a pair of singlet scalars $\phi$ if $2 m_{\phi}<m_{h}$, whereas it can decay into a pair of singlet neutrinos $S$ if $2 m_{S}<m_{h}$. The invisible Higgs decay widths are given at tree level by

$$
\begin{array}{rlrl}
\Gamma_{h \rightarrow \phi \phi} & =\frac{\left(\lambda v_{h} c+2 \lambda_{3} s v_{\Phi}\right)^{2}}{32 \pi m_{h}}\left(1-\frac{4 m_{\phi}^{2}}{m_{h}^{2}}\right)^{1 / 2} \quad & \left(\text { for } 2 m_{\phi}<m_{h}\right), \\
\text { and } \quad \Gamma_{h \rightarrow S S}=\frac{\left(Y_{\Phi} s\right)^{2} m_{h}}{8 \pi}\left(1-\frac{4 m_{S}^{2}}{m_{h}^{2}}\right)^{3 / 2} \quad & \left(\text { for } 2 m_{S}<m_{h}\right) .
\end{array}
$$

If the Higgs boson mass $m_{h}$ is smaller than $2 m_{\phi(S)}$, then the singlet particles can not be produced by real Higgs decays, but arise only via virtual Higgs exchange. We notice that since any produced singlet particles are not expected to interact inside the collider, they only give rise to strong missing energy signals.

To quantify the signals for the invisible decay of the Higgs boson, we investigate the ratio $R$ defined as follows [13]:

$$
R=\frac{\mathcal{B}_{h \rightarrow W^{+} W^{-}, Z Z, \bar{b} b, \bar{c} c, \bar{\tau} \tau}(\mathrm{SM}+\phi, S)}{\mathcal{B}_{h \rightarrow W^{+} W^{-}, Z Z, \bar{b} b, \bar{c} c, \bar{\tau} \tau}(\mathrm{SM})}=\frac{\Gamma_{h, \text { total }}(\mathrm{SM})}{\Gamma_{h \rightarrow \phi \phi(S S)}+\Gamma_{h, \text { total }}(\mathrm{SM})} .
$$

The ratio $R$ indicates how the expected signal for the visible decay of the Higgs boson can decrease due to the existence of the singlet scalar field $\phi$ and/or neutrino $S$. For $2 m_{\phi}>m_{h}$, 

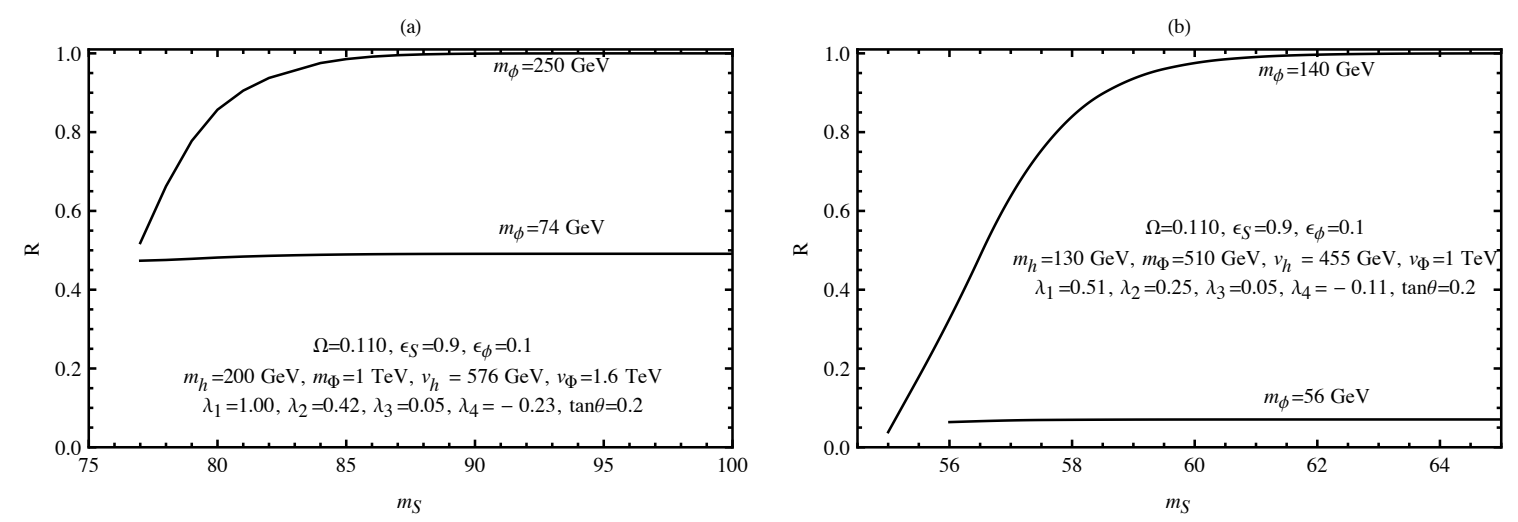

FIG. 5: Plots of the ratio $\mathrm{R}$ as a function of $m_{S}$ for our new model with $\Omega h^{2}=0.110$ for (a) $\varepsilon_{S}=1$, $\varepsilon_{\phi}=0, m_{h}=200 \mathrm{GeV}$ and (b) $\varepsilon_{S}=0.9, \varepsilon_{\phi}=0.1, m_{h}=130 \mathrm{GeV}$. The upper curves correspond to $m_{\phi}=$ (a) $120 \mathrm{GeV}$ and (b) $90 \mathrm{GeV}$. The lower curves correspond to $m_{\phi}=$ (a) $90 \mathrm{GeV}$ and (b) $58 \mathrm{GeV}$.

the decay mode $h \rightarrow \phi \phi$ is forbidden, so the ratio $R$ defined in Eq. (19) becomes

$$
\begin{aligned}
R & =\frac{\Gamma_{h, \text { total }}(\mathrm{SM})}{\Gamma_{h, \text { total }}(\mathrm{SM})}=1 & \left(2 m_{S} \geq m_{h}\right) \\
& =\frac{\Gamma_{h, \text { total }}(\mathrm{SM})}{\Gamma_{h \rightarrow S S}+\Gamma_{h, \text { total }}(\mathrm{SM})} & \left(2 m_{S}<m_{h}\right) .
\end{aligned}
$$

On the other hand, for $2 m_{\phi}<m_{h}$, the decay mode $h \rightarrow \phi \phi$ is allowed, so the ratio $R$ is given by

$$
\begin{aligned}
R & =\frac{\Gamma_{h, \text { total }}(\mathrm{SM})}{\Gamma_{h \rightarrow \phi \phi}+\Gamma_{h, \text { total }}(\mathrm{SM})} & \left(2 m_{S} \geq m_{h}\right) \\
& =\frac{\Gamma_{h, \text { total }}(\mathrm{SM})}{\Gamma_{h \rightarrow S S}+\Gamma_{h \rightarrow \phi \phi}+\Gamma_{h, \text { total }}(\mathrm{SM})} & \left(2 m_{S}<m_{h}\right)
\end{aligned}
$$

In Fig. 5, we present the ratio $R$ as a function of $m_{S}$ for the cases (a) $m_{\phi}=250$ (74) GeV and $m_{h}=200 \mathrm{GeV}$, (b) $m_{\phi}=140$ (56) $\mathrm{GeV}$ and $m_{h}=130 \mathrm{GeV}$, which would be probed right after LHC starts to run. The the upper and the lower curves in each panels of Fig. 5 correspond to $m_{h}<2 m_{\phi}$ and $m_{h}>2 m_{\phi}$, respectively. To estimate the ratio $R$, we need to specify some of unknown parameters: $\Omega h^{2}=0.110$ with $\lambda_{3}=0.05, \varepsilon_{S}=0.9, \varepsilon_{\phi}=0.1$. The upper limit of $m_{S}=100(65) \mathrm{GeV}$, as shown in Fig. 5, is adopted from the condition $2 m_{S}<m_{h}$. Note that the upper curves corresponding to a case for $2 m_{\phi}>m_{h}$ indicate that the scenario of $2 \mathrm{DM}$ can be probed via the ratio $R$ through the missing energy signal only for $m_{S}<m_{h} / 2$, i.e. (a) $77 \mathrm{GeV}<m_{S}<100 \mathrm{GeV}$ and (b) $55 \mathrm{GeV}<m_{S}<65 \mathrm{GeV}$. The lower 
curves corresponding to a case for $2 m_{\phi}<m_{h}$ show that the ratio $R$ is less than $\sim 0.5$ in (a) and $\sim 0.07$ in (b) for all possible values of $m_{S}$. The reason why the value $R$ is saturated by 0.5 in (a) and 0.07 in (b) (even for $2 m_{S}>m_{h}$ ) is that the decay mode $h \rightarrow \phi \phi$ dominates over the decay mode $h \rightarrow S S$ in this case (See Eq. (21)). The lower curve indicates that we can rather easily probe the $2 \mathrm{DM}$ scenario by measuring the ratio $R$ as long as $2 m_{\phi}<m_{h}$.

\section{CONCLUSION}

We have examined how the scenario of coexisting two-particle dark mater can be realized in the extended seesaw model, which has been previously proposed to accommodate small neutrino masses and low scale leptogenesis. In this scenario, we impose the discrete symmetry $Z_{2} \times Z_{2}^{\prime}$ and introduce new renormalizable interaction terms containing the heavy singlet scalar particle $\Phi$ so as for singlet Majorana neutrino $S$ and singlet scalar $\phi$ to be the coexisting two dark matter candidates. Depending on the mass spectrum of the two dark matter candidates, the annihilation processes either $S S \longrightarrow \phi \phi$ or $\phi \phi \longrightarrow S S$ is of particular interest because the annihilation cross sections for the processes can be so large that the relic abundance of decaying particle should get lowered, which in turn makes the constraints on its parameter space relaxed, compared with the case of one and only one dark matter candidate. We have also discussed the implications of the dark matter detection through the scattering off the nucleus of the detecting material on our scenarios for dark matter candidate. Our results show that the expected reach of SuperCDMS could detect a signal for dark matter or at least strongly constrain the parameter space, while the recent result of XENON10 Dark Matter experiment does not so. In addition, we have studied the implications for the search of invisible Higgs decay at LHC which may serve as a probe of our scenarios for dark matter. In particular, we have found that there is a chance to easily probe the $2 \mathrm{DM}$ scenario by measuring the ratio $R$ as long as $2 m_{\phi}<m_{h}$.

Acknowledgement: SKK is supported by the KRF Grant funded by the Korean Government(MOEHRD) (KRF-2006-003-C00069). CSK and HSC are supported in part by CHEP-SRC and in part by the Korea Research Foundation Grant funded by the Korean Government (MOEHRD) No. KRF-2005-070-C00030. 
[1] J. Dunkley et al. [WMAP Collaboration], arXiv:0803.0586 [astro-ph].

[2] G. R. Blumenthal, S. M. Faber, J. R. Primack and M. J. Rees, Nature 311, 517 (1984); L. Bergstrom, Rept. Prog. Phys. 63, 793 (2000) arXiv:hep-ph/0002126]; V. Sahni, Lect. Notes Phys. 653, 141 (2004) arXiv:astro-ph/0403324.

[3] G. Jungman, M. Kamionkowski and K. Griest, Phys. Rept. 267, 195 (1996) |arXiv:hep-ph/9506380]; G. Bertone, D. Hooper and J. Silk, Phys. Rept. 405, 279 (2005) arXiv:hep-ph/0404175.

[4] G. Steigman and M. S. Turner, Nucl. Phys. B 253, 375 (1985); B. W. Lee and S. Weinberg, Phys. Rev. Lett. 39, 165 (1977); S. Weinberg, Phys. Rev. Lett. 48, 1303 (1982).

[5] http://xenon.physics.rice.edu/xenon100.html

[6] R. W. Schnee et al. [The SuperCDMS Collaboration], arXiv:astro-ph/0502435; P. L. Brink et al. [CDMS-II Collaboration], In the Proceedings of 22nd Texas Symposium on Relativistic Astrophysics at Stanford University, Stanford, California, 13-17 Dec 2004, pp 2529 arXiv:astro-ph/0503583; D. S. Akerib et al., Nucl. Instrum. Meth. A 559, 411 (2006); R. W. I. Ogburn, In the Proceedings of International Symposium on Detector Development for Particle, Astroparticle and Synchrotron Radiation Experiments (SNIC 2006), Menlo Park, California, 3-6 Apr 2006, pp 0150; T. Bruch [CDMS Collaboration], AIP Conf. Proc. 957, 193 (2007).

[7] Q. H. Cao, E. Ma, J. Wudka and C. P. Yuan, arXiv:0711.3881 [hep-ph].

[8] A. Palazzo, D. Cumberbatch, A. Slosar and J. Silk, Phys. Rev. D 76, 103511 (2007) arXiv:0707.1495 [astro-ph]].

[9] H. Sung Cheon, S. K. Kang and C. S. Kim, JCAP 0805, 004 (2008), arXiv:0710.2416 [hep-ph].

[10] S. K. Kang and C. S. Kim, Phys. Lett. B 646, 248 (2007) arXiv:hep-ph/0607072;

S. K. Kang and C. S. Kim, Int. J. Mod. Phys. E 16 (2007) 1505.

[11] M. C. Bento, O. Bertolami, R. Rosenfeld, L. Teodoro, Phys. Rev. D62, 041302 (2000) arXiv:astro-ph/0003350];M. C. Bento, O. Bertolami, R. Rosenfeld, Phys. Lett. B518, 276 (2001) arXiv:hep-ph/0103340;G. Cynolter, E. Lendvai and G. Pocsik, Acta Phys. Polon. B 36, 827 (2005) arXiv:hep-ph/0410102 ; K. S. Babu and E. Ma, Int. J. Mod. Phys. A 
23, 1813 (2008) arXiv:0708.3790 [hep-ph]]; Y. G. Kim and K. Y. Lee, Phys. Rev. D 75, 115012 (2007) arXiv:hep-ph/0611069; Y. G. Kim, K. Y. Lee and S. Shin, JHEP 0805, 100 (2008) arXiv:0803.2932 [hep-ph]]; M. M. Ettefaghi and M. Dehghani, arXiv:0805.0682 [hep-ph]; H. Davoudiasl, R. Kitano, T. Li and H. Murayama, Phys. Lett. B 609, 117 (2005) arXiv:hep-ph/0405097]; V. Barger, P. Langacker, M. McCaskey, M. J. Ramsey-Musolf and G. Shaughnessy, Phys. Rev. D 77, 035005 (2008) [arXiv:0706.4311 [hep-ph]]; R. Dick, R. B. Mann and K. E. Wunderle, Nucl. Phys. B 805, 207 (2008) arXiv:0803.1444 [astro-ph]].

[12] D. N. Spergel and P. J. Steinhardt,, Phys. Rev. Lett. 84, 3760 (2000) arXiv:astro-ph/9909386]; C. Firmani, E. D'Onghia, V. Avila-Reese, G. Chincarini and X. Hernandez, Mon. Not. Roy. Astron. Soc. 315, (2000) L29; B. D. Wandelt, R. Dave, G. R. Farrar, P. C. McGuire, D. N. Spergel and P. J. Steinhardt, arXiv:astro-ph/0006344 R. Dave, D. N. Spergel, P. J. Steinhardt and B. D. Wandelt, Astrophys. J. 547, 574 (2001) arXiv:astro-ph/0006218; A. Kusenko and P. J. Steinhardt, Phys. Rev. Lett. 87, 141301 (2001) arXiv:astro-ph/0106008; J. McDonald, Phys. Rev. Lett. 88, 091304 (2002) arXiv:hep-ph/0106249 ; J. E. Lidsey, T. Matos and L. A. Urena-Lopez, Phys. Rev. D66, 023514 (2002) arXiv:astro-ph/0111292]; S. Balberg, S. L. Shapiro and S. Inagaki, Astrophys. J. 568, 475 (2002) arXiv:astro-ph/0110561; A. E. Faraggi and M. Pospelov, Astropart. Phys. 16, (2002) 451 arXiv:hep-ph/0008223; A. L. Erickcek, P. J. Steinhardt, D. McCammon and P. C. McGuire, Phys. Rev. D76, 042007 (2007) arXiv:0704.0794 [astro-ph]; A. K. Ganguly, P. Jain, S. Mandal and S. Stokes, Phys. Rev. D76, 025026 (2007) arXiv:hep-ph/0611006; G. D. Mack, J. F. Beacom and G. Bertone, Phys. Rev. D76, 043523 (2007) arXiv:0705.4298 [astro-ph].

[13] J. McDonald, Phys. Rev. D 50, 3637 (1994) arXiv:hep-ph/0702143]; C.P.Burgess, Maxim Pospelov, Tonnis ter Veldhuis, Nucl. Phys. B619, 709 (2001) arXiv:hep-ph/0011335.

[14] Kim Griest, David Seckel, Phys. Rev. D43, 3191 (1991).

[15] G. Belanger, F. Boudjema, A. Pukhov and A. Semenov, Comput. Phys. Commun. 177, 894 (2007).

[16] J. Angle et al., arXiv:0706.0039 [astro-ph].

[17] D. S. Akerib et al. [CDMS Collaboration], Phys. Rev. Lett. 96, 011302 (2006) arXiv:astro-ph/0509259.

[18] See also, K. Belotsky, D. Fargion, M. Khlopov, R. Konoplich and K. Shibaev, Phys. Rev. D 68, 054027 (2003) arXiv:hep-ph/0210153. 